\title{
Materials and Life Science with Quantum Beams at the Japan Proton Accelerator Research Complex
}

\author{
Klaus-Dieter Liss ${ }^{1,2}$ (D) \\ 1 Guangdong Technion - Israel Institute of Technology, Shantou 515063, China; kdl@gtiit.edu.cn or \\ kdliss@technion.ac.il or liss@kdliss.de; Tel.: +86-0754-8807-7102 \\ 2 Technion - Israel Institute of Technology, Haifa 32000, Israel
}

Received: 7 May 2018; Accepted: 14 May 2018; Published: 14 May 2018

\section{Introduction}

The Materials and Life Science Experimental Facility (MLF) is the major neutron and muon quantum beam facility in the eastern part of the world. Besides the neutrino experiment and the hadron facility, it forms part of the Japan Proton Accelerator Research Complex (J-PARC), a scientific landmark installation pictured in Figure 1. The MLF delivers quantum beams to 20 neutron beamlines, plus one under commissioning, and two muon beamlines hosting three end-stations. Figure 2 displays a schematic layout, and an impressive view into one of the experimental halls is shown in Figure 3. The first neutron and muon beams were produced in 2008. Nowadays, the facility serves about 700 peer-reviewed and accepted proposals per year by users coming from 34 countries across the globe. Applications range from engineering to life science, include soft and hard condensed matter and consider cryogenic conditions, high temperatures, and extreme pressure. MLF is a division of J-PARC governed by a consortium of the Japan Atomic Energy Agency (JAEA) [1] and the High Energy Accelerator Research Organization, KEK [2], as stakeholders. User promotion services and operation of the seven MLF public-access facilities are codirected by the Comprehensive Research Organization for Science and Society (CROSS) [3], not only cooperating closely with the J-PARC Users Office but also providing supporting scientific manpower, safety and scientific management.

The book on Materials and Life Science with Quantum Beams at the Japan Proton Accelerator Research Complex is an extract of the larger Topical Collection Facilities in the journal Quantum Beam Science [4], compiling a comprehensive description of MLF at J-PARC. Interestingly, the concept of Quantum Beam Science reunifying various short-wavelength and high-energetic electromagnetic and particle beams, predominantly for applications in materials and life sciences [5], has been minted in Japan around the stakeholder organizations [6]. It strongly recognizes the need for various quantum beams for the investigation of peculiar materials science problem. Accordingly, MLF operates two major quantum beam sources-neutrons and muons. Complementary access at Japan's major synchrotron SPring- 8 and other large-user facilities is strongly supported in the beam-time application and reviewing processes.

\section{Overview and Chapters}

The book has been separated into four review articles, as published in the journal Quantum Beam Science [8-11]. Central to all J-PARC experimental divisions is the landmark accelerator complex (Figure 1), composed of a linear accelerator (LINAC) feeding $\mathrm{H}^{-}$ions by stripping them to protons into the Rapid Cycle Synchrotron. Further accelerated to $3 \mathrm{GeV}$, those protons are used to produce the muon and neutron sources at MLF. Besides feeding MLF, protons can be further accelerated to $50 \mathrm{GeV}$ in the main accelerator ring, serving the Hadron Beam Facility for creating pions, kaons and other hadrons for fundamental nuclear and particle physics, including the higher generation quark families, such as the strange and charm quarks [12]. The neutrino facility is another experiment of 
global character, producing light-mass particle radiation in an underground station with detectors both nearby and $295 \mathrm{~km}$ across Honshu island at the Super-Kamiokande detector, chasing muon-neutrino oscillations to probe the most fundamental coupling constants of the universe [13]. The scope of the present book, however, focuses on MLF and its neutron and muon beams as applied to a vast range of disciplines based on the material and life sciences. Their central layout is given in Figure 2, with respective sources and beamlines, occupying buildings of hundred-meter dimensions and vast experimental halls, Figure 3.

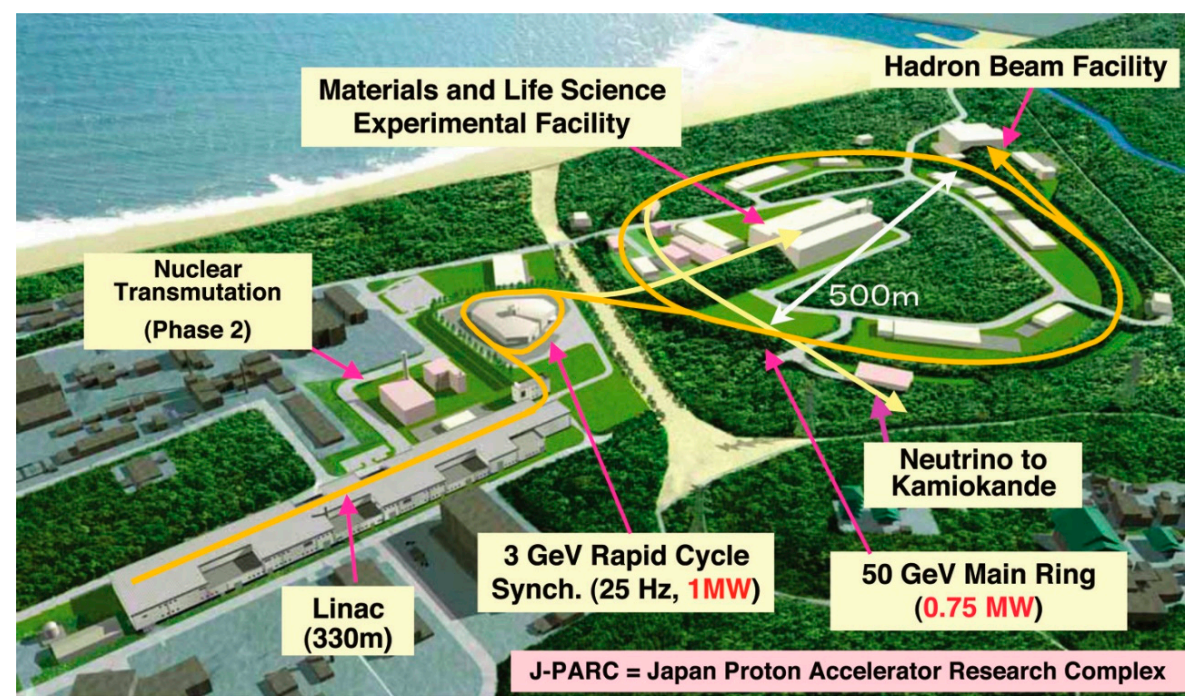

Figure 1. Schematic aerial view of the entire J-PARC complex. To the left is the linear accelerator (LINAC), feeding protons into the rapid cycle synchrotron. Once accelerated to $3 \mathrm{GeV}$, they are delivered to the Materials and Life Sciences facility, or alternatively further accelerated to $50 \mathrm{GeV}$ and delivered to one of the other two large laboratories: the hadron facility and the neutrino experiment. (Modified from “The entire view of J-PARC" by Shoji Nagamiya and published under a CC BY-SA 3.0 license [7]).

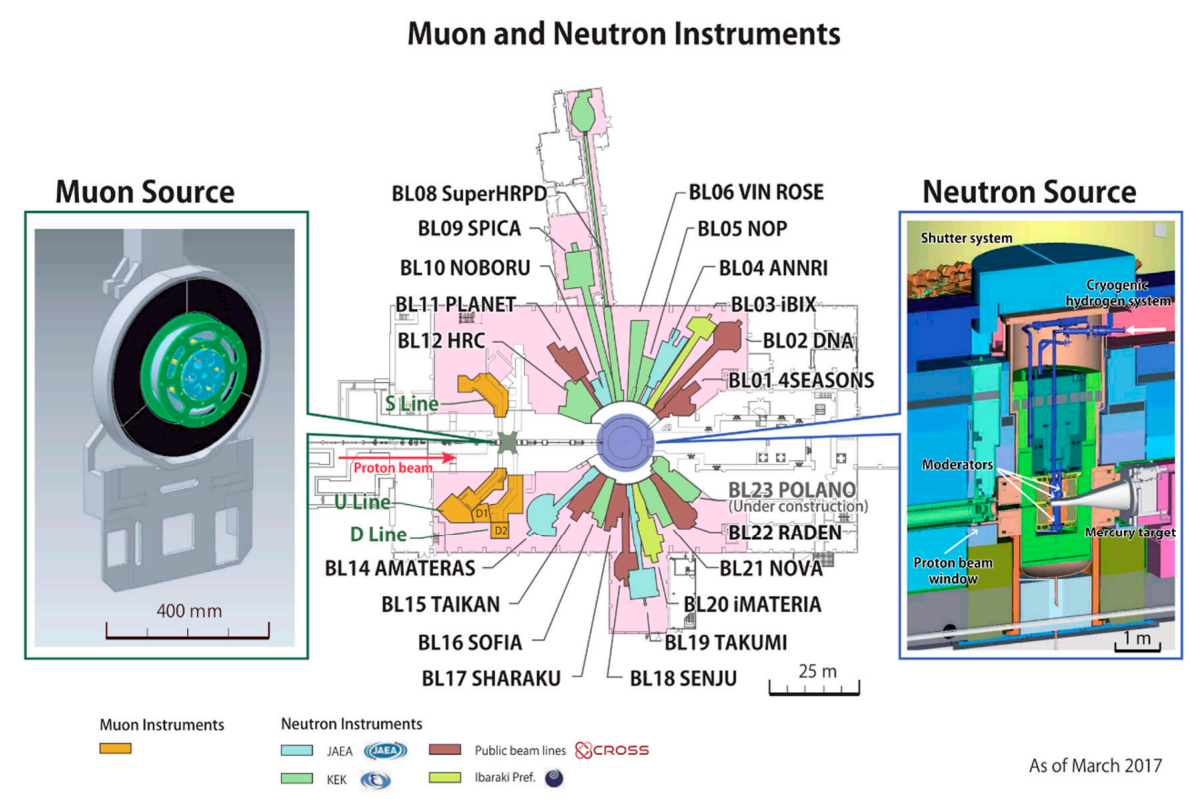

Figure 2. Layout of the muon and neutron beamlines (center) with drawings of the sources (left and right). The color codes of the neutron beamlines denote the operator organizations as per legend. (Reproduced from [14] with permission). JAEA: Japan Atomic Energy Agency; KEK: High Energy Accelerator Research Organization; CROSS: Comprehensive Research Organization for Science and Society. 


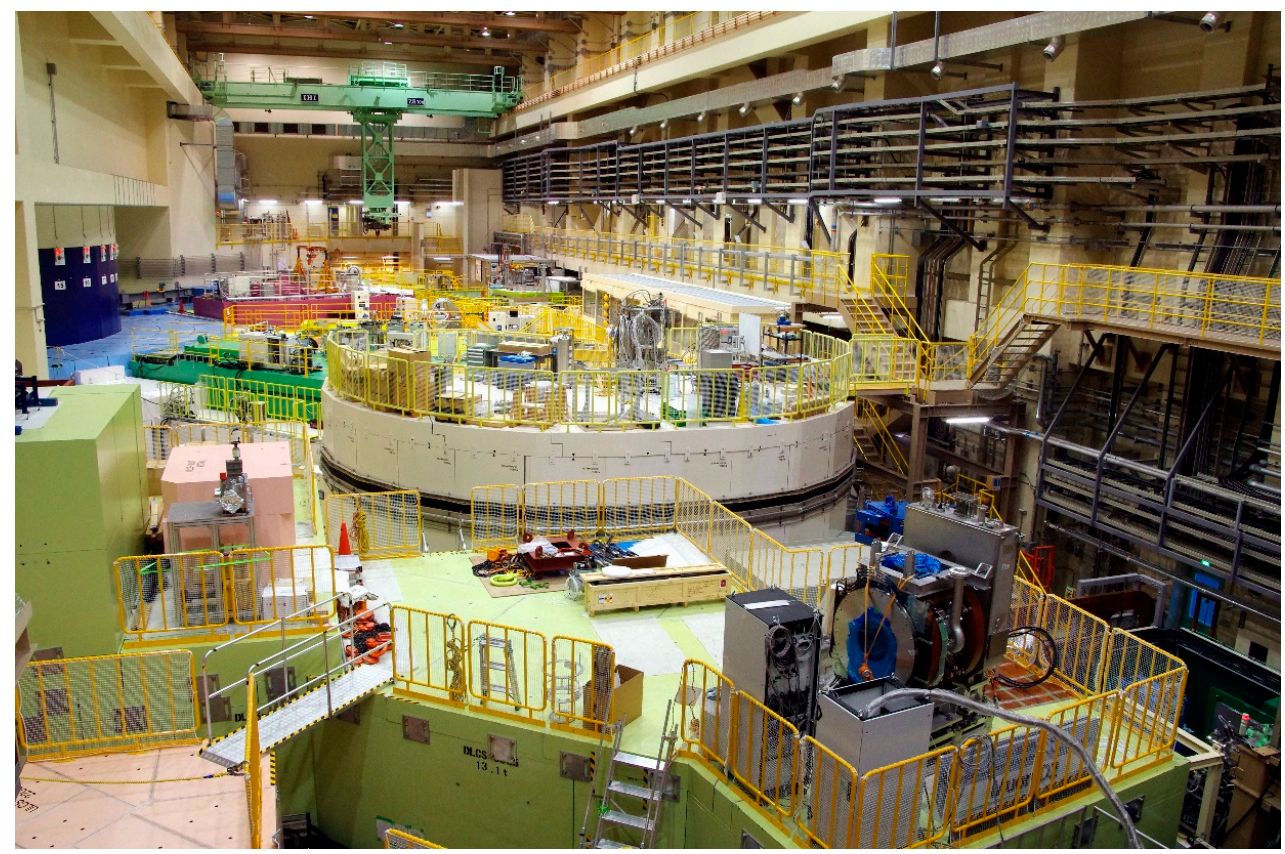

Figure 3. Photograph of the Materials and Life Science Experimental Facility (MLF) Experimental Hall II at J-PARC with the muon D-line shielding in the foreground and looking over the neutron beamlines BL14 in the image center to BL23 at the far back (compare to Figure 2).

The four sections are divided into descriptions of the Pulsed Spallation Neutron Source [8], the Neutron Scattering Instruments [9], the Neutron Devices and Computational and Sample Environments [10], and the Muon Facility [11].

\subsection{Pulsed Spallation Source}

The core of the neutron beam lines is the pulsed spallation source reviewed in the article by Takada et al. [8]. The target is the device where the accelerated proton beam hits heavy atoms, and spallation takes place by excitation of the nuclei and subsequent evaporation of neutrons. Moreover, time-of-flight neutron analysis is used throughout the beamline facilities, requiring well-defined neutron pulses. Spallation at the liquid mercury target produces high-energy neutrons, which need to be moderated into the desired thermal and cold neutron spectral distributions. Proton beam pulse shape, the spallation process, location and optics of the moderators, and type of instrument determine the neutron pulse shape, which is essential to optimize instrument resolution. The proton beam power is designed up to $1 \mathrm{MW}$, rendering the engineering technology of the target and moderators critical for handling mechanical shock waves, cryogenic cooling, and radiation damage. The review describes in detail such engineering solutions and the performance of the beams.

\subsection{Neutron Scattering Instruments}

The main focus of a user of the neutron facility is on the kind and capabilities of beamlines and neutron scattering instruments, reviewed here by Nakajima et al. [9]. The 21 dedicated and unique beamlines are grouped according to their methods and applications into (i) Spectroscopy, (ii) Crystal-Structure, (iii) Nano-Structure and (iv) Pulsed Neutron Application.

Crystal structures (ii) are determined by the neutron diffraction method, obeying elastic momentum transfer by Bragg's law or the Laue equation. The differences and peculiarities of the seven beamlines lie in extra-high resolution, and sample environments for classes of materials-such as engineering, chemistry, biological crystals, or specimens under extreme conditions. 
On top of a potential diffraction component, spectroscopy (i) deals with inelastic scattering processes where the neutron loses or gains energy due to interaction with lattice vibrations-phonons, diffusion, potential-barrier tunneling, or other dynamic and kinetic processes occurring in the specimen. As with the diffraction group, the six instruments vary according to applications and specifications. The latest instrument under commissioning is a polarized neutron spectrometer, sensitive to magnetic and spin excitations in solid materials.

Objects larger than atomic and basic crystal structures, such as micelles, cells, precipitates, polymers, lipids, foams, hierarchical structures, domains, quantum dots, amorphous materials, thin films, etc. scatter on the 'small angle' or 'small momentum transfer' small-Q scale, forming the nano-structure group (iii) by probing nanometer length scales. The four dedicated beamlines extend over small-Q bulk scattering, (polarized) reflectometry for (magnetic) thin film and interface studies as well as instruments covering the overlap from small-Q to the conventional diffraction regime, and the measurement of pair distribution functions.

Finally, fundamental neutron physics, neutron optics and the important field of neutron imaging are grouped into the pulsed neutron applications (iv). Here the instrumentation takes particular advantage of the pulsed neutron bunches, such as the recent technique development of energy-resolved neutron imaging, where Bragg edge diffraction is used in transmission radiography to obtain or discriminate the signal by crystallographic information and obtain additional contrast.

Altogether, it can be stated that J-PARC/MLF has developed and constructed world-class, state-of-the-art beamlines covering all aspects of neutron scattering with respect to complementary and combined, multi-dimensional or time-resolved data acquisition, serving a variety of disciplines and sample systems.

\subsection{Neutron Devices and Computational and Sample Environments}

The aforementioned beamlines, coverage of disciplines, length and time scales, specimen conditions and multi-dimensional acquisition methods require technological development of devices, computational data acquisition and instrument control, and special sample environments, described here by Sakasai et al. [10]. There exist a multitude of optical and timing devices-such as focusing mirrors, neutron guides, choppers at one end-matched by a scattering geometry with sample handling and a secondary spectrometer to the detector. As each beamline is unique with very specific requirements, so are those devices.

Each beamline may consist of hundreds to thousands of detector channels of which each may be divided into a neutron time-of-flight pattern; each detector angle may be different to its neighbor pixel, recording different time-of-flight patterns that finally have to be calibrated and mapped into physically meaningful, sample-specific diffractograms and energy-transfer spectra. The measured dimensions can be high as three-dimensional reciprocal space plus energy resolution, represented in four dimensions. Similar dimensionality or higher is obtained in three-dimensional computed tomography with reciprocal space and time information in addition.

MLF instruments are among the first neutron scattering facilities to have implemented so called event-mode, in which a time stamp for each registered neutron is recorded, allowing to re-bin histograms after the experiment from the stored data. Such practice necessitates not only huge storage capacities but also computing algorithms and user interfaces, which have been developed and are presented.

Last but not least, although special sample environments such as high-pressure devices were described in the previous chapter, their electronics and integration demands special considerations, as reported in the same chapter.

\subsection{The Muon Facility}

The chapter by Higemoto et al. [11] describes the muon facility, which at first sight appears distinct from the neutron facility, however it is complementary for some user communities, e.g., for the determination of magnetic structure and properties of a material. Not only do the operation of the 
sources show synergies, but there are also similarities in data acquisition systems and infrastructure. Since the user community and physical installation are much smaller than for neutron scattering, the article reviews all aspects from the source, beamlines and applications. Similarly, the flux and energy of muons vary. For example, in conventional spectroscopy where a muon is embedded in the bulk of a material, its decay time and behavior reveal atomic local structural properties. Or moreover, the conditioning of ultra-slow muons with particular low kinetic energies can specifically probe the surface of a specimen. Examples of applications are reviewed on a new antiferromagnetic phase in iron-based superconductor, magnetic ground states of iridium spinel compound, or non-invasive element analysis by muonic $\mathrm{X}$-ray spectroscopy.

\section{Conclusions and Outlook}

With a unique park of instrumentation and technological development, 10 years after first beam MLF at J-PARC is one of the world-leading facilities, serving over 700 proposals per year. It is one of the three major spallation neutron sources operating and one of the four muon facilities worldwide, and has potential for further development in both beam power and instrumentation. While the last neutron beamline slots are being occupied and commissioned, effort is focused on dedicated sample environments which are second to none in the world.

I would like to express my congratulations to the facility and invite the readers and experts to enjoy the chapters of the present book on the J-PARC Materials and Life Science Experimental Facility for reference, documentation and support of their research and development.

Conflicts of Interest: The author declares no conflict of interest.

\section{References}

1. Japan Atomic Energy Agency. Available online: https://www.jaea.go.jp/english/ (accessed on 30 April 2018).

2. KEK, High Energy Accelerator Research Organization. Available online: http://www.kek.jp/en/ (accessed on 30 April 2018).

3. CROSS Neutron Science and Technology Center. Available online: https://neutron.cross.or.jp/en (accessed on 30 April 2018).

4. Liss, K.-D. Facilities in Quantum Beam Science. Quantum Beam Sci. 2018, 2, 6. [CrossRef]

5. Liss, K.-D. Quantum Beam Science-Applications to Probe or Influence Matter and Materials. Quantum Beam Sci. 2017, 1, 1. [CrossRef]

6. Yamamoto, H.; Igawa, N.; Moriai, A.; Sakashita, T.; Ohba, H.; Sekiguchi, T.; Yasuda, R.; Kawano, T.; Suzuki, E. (Eds.) Annual Report QuBS 2013; Quantum Beam Science Directorate, Japan Atomic Energy Agency: Tokai, Japan, 2014.

7. Nagamiya, S. Introduction to J-PARC. Prog. Theor. Exp. Phys. 2012, 2012, 020001. [CrossRef]

8. Takada, H.; Haga, K.; Teshigawara, M.; Aso, T.; Meigo, S.-I.; Kogawa, H.; Naoe, T.; Wakui, T.; Ooi, M.; Harada, M.; et al. Materials and Life Science Experimental Facility at the Japan Proton Accelerator Research Complex I: Pulsed Spallation Neutron Source. Quantum Beam Sci. 2017, 1, 8. [CrossRef]

9. Nakajima, K.; Kawakita, Y.; Itoh, S.; Abe, J.; Aizawa, K.; Aoki, H.; Endo, H.; Fujita, M.; Funakoshi, K.; Gong, W.; et al. Materials and Life Science Experimental Facility (MLF) at the Japan Proton Accelerator Research Complex II: Neutron Scattering Instruments. Quantum Beam Sci. 2017, 1, 9. [CrossRef]

10. Sakasai, K.; Satoh, S.; Seya, T.; Nakamura, T.; Toh, K.; Yamagishi, H.; Soyama, K.; Yamazaki, D.; Maruyama, R.; Oku, T.; et al. Materials and Life Science Experimental Facility at the Japan Proton Accelerator Research Complex III: Neutron Devices and Computational and Sample Environments. Quantum Beam Sci. 2017, 1, 10. [CrossRef]

11. Higemoto, W.; Kadono, R.; Kawamura, N.; Koda, A.; Kojima, K.M.; Makimura, S.; Matoba, S.; Miyake, Y.; Shimomura, K.; Strasser, P. Materials and Life Science Experimental Facility at the Japan Proton Accelerator Research Complex IV: The Muon Facility. Quantum Beam Sci. 2017, 1, 11. [CrossRef]

12. Noumi, H. Strange and Charm Hadron Physics at J-PARC in Future. In Proceedings of the 12th International Conference on Hypernuclear and Strange Particle Physics (HYP2015), Sendai, Japan, 7-12 September 2015. 
13. Abe, K.; Abgrall, N.; Aihara, H.; Ajima, Y.; Albert, J.B.; Allan, D.; Amaudruz, P.-A.; Andreopoulos, C.; Andrieu, B.; Anerella, M.D.; et al. The T2K experiment. Nucl. Instrum. Methods Phys. Res. Sect. A Accel. Spectrom. Detect. Assoc. Equip. 2011, 659, 106-135. [CrossRef]

14. Kojima, K.M.; Kawamura, N.; Mishima, K.; Naoe, T.; Oikawa, K.; Parker, J.; Sakasai, K.; Tominaga, T.; Tanaka, K. (Eds.) J-PARC MLF Annual Report 2016; J-PARC: Tokai, Japan, 2017. 